\title{
Flaring and self-shadowed disks around Herbig Ae stars: simulations for $10 \mu \mathrm{m}$ interferometers
}

\author{
R. van Boekel ${ }^{1,2}$, C. P. Dullemond ${ }^{3}$, and C. Dominik ${ }^{1}$ \\ 1 Sterrenkundig Instituut "Anton Pannekoek", Kruislaan 403, 1098 SJ Amsterdam, The Netherlands \\ e-mail: vboekel@science.uva.nl \\ 2 European Southern Observatory, Karl-Schwarzschild-Strasse 2, 85748 Garching, Germany \\ 3 Max-Planck-Institut für Astronomie Heidelberg, Königstuhl 17, Heidelberg, Germany
}

Received 26 October 2004 / Accepted 27 June 2005

\begin{abstract}
We present simulations of the interferometric visibilities of Herbig Ae star disks. We investigate whether interferometric measurements in the $10 \mu \mathrm{m}$ atmospheric window are sensitive to the presence of an increased scale height at the inner disk edge, predicted by recent models. Furthermore, we investigate whether such measurements can discriminate between disks with a "flaring" geometry and disks with a "flat" geometry. We show that both these questions can be addressed, using measurements at a small number of appropriately chosen baselines. The classification of Herbig Ae stars in two groups, based on the appearance of the spectral energy distribution (SED), has been attributed to a difference in disk geometry. Sources with a group I SED would have a flaring outer disk geometry, whereas the disk of group II sources is proposed to be flat (or "self-shadowed"). We show that this hypothesis can be tested using long-baseline interferometric measurements in the $10 \mu \mathrm{m}$ atmospheric window.
\end{abstract}

Key words. stars: circumstellar matter - stars: pre-main-sequence - techniques: interferometric - radiative transfer

\section{Introduction}

Herbig Ae/Be stars (HAEBEs, Herbig 1960; for a more recent review see Natta et al. 2000) are intermediate mass premain-sequence stars, surrounded by material which is left from the star formation process. A sub-group of mostly late B and A-F type HAEBE stars (hereafter HAEs) show little or no optical extinction, and usually have low mass accretion rates as derived from radio analysis (Skinner et al. 1993) and the lack of significant veiling in optical spectra. There is ample evidence that the circumstellar material responsible for the large infrared excesses of these stars is located in a circumstellar disk (e.g. Mannings \& Sargent 1997; Grady et al. 2001; Augereau et al. 2001; Eisner et al. 2003). Vink et al. (2002) show that the gaseous component has a disk-like geometry on scales of less than $0.1 \mathrm{AU}$.

Whereas the presence of these circumstellar disks seems firmly established, the structure of the disks is a matter of debate. Kenyon \& Hartmann (1987) developed "flaring" disk models for T-Tauri stars, in which $H / R$ (the ratio of the disk surface height to the distance to the star) increases with increasing distance to the central star. The flaring disk model was refined by Chiang \& Goldreich (1997, hereafter CG97) who introduced an optically thin surface layer responsible for the infrared emission features generally seen in circumstellar disks. Natta et al. (2001) and Dullemond et al. (2001) reconsider the CG97 model in the context of HAe stars, proposing that the innermost region of the disk has an increased scale height: the "puffed-up inner rim". This configuration, which results from hydrostatic equilibrium at the directly irradiated inner rim, naturally explains the near-infrared bump commonly observed in the Spectral Energy Distribution (SED) of HAe systems (Natta et al. 2001).

Meeus et al. (2001) noted that based on the IR SED, HAEs can be divided into two main groups: "group I" sources that have a very strong, rising IR excess peaking around $60 \mu \mathrm{m}$, and "group II" sources displaying a more moderate IR excess, lacking the $60 \mu \mathrm{m}$ bump. It was proposed that group I sources have a "flaring" geometry, allowing the disk to intercept and reprocess stellar radiation out to large stellocentric radii. In the outer disk of group II sources, on the other hand, $H / R$ is approximately constant, or decreasing with increasing distance to the star. The inner disk shields the outer disk from direct irradiation by the central star, hence the term "self-shadowed" disk. This substantially reduces the amount of radiation absorbed locally, leading to lower temperatures in the outer disk of a group II source.

Recent 2D modeling by Dullemond (2002), Dullemond \& Dominik (2004a, henceforth DD04), has quantitatively confirmed that both flaring and self-shadowed disks are natural solutions of the equation of vertical hydrostatic equilibrium in passive circumstellar disks (see also Sect. 2.4). These models form the basis of the current study. There is ample evidence that group I and group II disks indeed have a flaring and 
self-shadowed geometry, respectively (e.g. Grady et al. 2004; Dullemond \& Dominik 2004b; Leinert et al. 2004). However, as this is not an observational study, we will consistently refer to the models as flaring/self-shadowed, rather than group I/II (which is by definition an SED classification).

With the advent of long $\left(\sim 10^{2} \mathrm{~m}\right)$ baseline infrared interferometry using large apertertures, it has now become possible to observe HAe disks in the thermal infrared with a spatial resolution of order $10^{-2}$ arcsec. At the present, the number of baselines will be limited, and only interferometric amplitudes (no phases) are available. True aperture synthetis imaging of disks is therefore not (yet) possible. The interpretation of the measured visibility amplitudes, which contain information about the geometry of the disks, requires the use of disk models.

In the near-infrared Herbig Ae/Be star disks have been observed with long-baseline interferometers since a number of years (Tuthill et al. 2001; Millan-Gabet et al. 2001; Eisner et al. 2003, 2004, 2005). Up to recently these measurements were evaluated using extremely simplified models: Gaussian blobs, rings, ellipses etc. Such simple models made it possible to get a handle on the typical size and inclination of the emitting source, but did not go much further. For most sources the typical sizes were found to be in rough agreement with those predicted by the inner rim models, but at high accretion rates the observations deviate from predictions. This is explained by Akeson et al. (2005) as due to the emission from accretion inward of the inner dust rim, and by Monnier et al. (2005) as due to the protection of dust by optically thick gas, allowing the dust to survive closer to the star. In these, and other, recent papers the modeling of the data already starts to go well beyond the simple ring/ellipse models, using actual multi-dimensional radiative transfer calculations in the case of Akeson et al., and detailed accretion disk structure models in the case of Lachaume et al. (2003). In particular with the new phase-closure capabilities in the near-infrared at the IOTA and VLTI/AMBER interferometers such more advanced models are clearly of great use.

With the mid-infrared interferometric capabilities of the MIDI instrument on the VLTI it is now possible to study the structure of the disk at slightly larger scales than the inner rim. This is the region in which the self-shadowed and flaring disks would most clearly be distinguishable. A first set of measurements was published by Leinert et al. (2004), and a first tentative correlation between the SED (group I/II) and the visibility was found. Mid-infrared interferometry also has the interesting capability of measureming mineralogical properties of the dust as a function of stellocentric radius. First measurements of this kind (van Boekel et al. 2004a) have revealed the strong radius-dependence of the crystallinity of dust, as predicted by theoretical models. In the present paper, however, we will be mostly concerned with the first aspect of mid-infrared interferometry: measuring the geometry of the disk.

Based on the 2D disk models of Dullemond \& Dominik (2004a) as well as the simpler models of Chiang \& Goldreich (1997) we present calculations of the interferometric visibilities of HAe disks, to investigate if it is possible to distinguish between the various disk geometries predicted by these theoretical models. Since the mid-infrared probes structure at somewhat larger scales than the inner rim (from $1 \mathrm{AU}$ out to about $20 \mathrm{AU}$ ), this wavelength regime is more suited to our aims than the near-infrared. The MIDI instrument is, so far, the only instrument capable of doing such measurements for Herbig Ae/Be stars, so in our analysis we focus on the typical baselines and properties of the VLTI.

\section{Modeling method}

The goal of this study is to predict and compare interferometric visibilities of various disk models. Synthetic disk images are made using a ray tracing algorithm, where special care is taken to ensure all spatial scales in the disk are sufficiently resolved. Interferometric visibilities are calculated by Fourier transforming the images.

\subsection{The inner rim}

In the disk models considered here, the bright inner rim is treated in a highly simplified fashion: it is a sharp, "vertical wall". As a consequence, when such a model is viewed not pole-on, the flux from the near side of the inner rim is strongly suppressed since the hot, irradiated rim surface is occulted by the cooler parts immediately outside the rim surface. The far side of the bright inner rim is in full view and is responsible for essentially all of the near-infrared excess. In this configuration, the total amount of near-infrared emission depends strongly on the disk inclination, suggesting that the observed strength of the " 3 micron bump" in the SED is a measure of the latter. Observations of Herbig Ae stars, however, show that they all have rather similar near-infrared excesses, irrespective of their inclination (Natta et al. 2000; Dominik et al. 2003).

This indicates that the appearance of the inner rim is more smooth than the "vertical wall" used here. The processes that determine the shape of the inner rim are currently not yet understood. Isella \& Natta (2005) recently showed that the dependence of the evaporation temperature on pressure naturally leads to a rounded-off inner rim. When such a disk is viewed at an inclination, both the near and the far side of the inner rim will be bright (although still the far side will be brighter). The bright inner rim will look like an inclined ring on the sky, rather than the "half ring" one obtains using the vertical wall model.

Realistic radiative transfer modeling of a rounded-off inner rim introduces various numerical complications. To avoid these difficulties we adopt the simplified vertical rim structure used in Dullemond \& Dominik (2004a). For a slightly off-polar inclination we artificially circularize the disk emission to circumvent the near-side/far-side asymmetry of the rim. In this way we mimic the rounded-off shape of the rim without having to confront the numerical complexities of radiative transfer in extreme optical depth rounded-off rims. While the spatial resolution of current $10 \mu \mathrm{m}$ interferometers is just sufficient to measure the diameter of the inner rim, observations at higher resolution are required to study details of the rim structure. We therefore believe that using this simplified approach is justified for our current purposes. To first approximation, inclination can be included by scaling the calculated spatial frequencies (or interferometric baselines) by a factor $1 / \cos (\theta)$ along the minor 
axis of an inclined disk, where $\theta$ is the inclination of the disk. At high inclinations, this approximation brakes down.

\subsection{Interferometry}

Rather than images, an interferometer produces an interference signal called the interferometric "visibility" $(V)$, which is the spatial coherence function of the intensity distribution of the source. The visibility is related to the intensity distribution through the van-Cittert-Zernike theorem, which states that the visibility is the Fourier transform of the intensity distribution of the source. For an introduction to long-baseline interferometry, we refer to Lawson (2000).

\subsection{Spectrally resolved visibilities}

Traditionally, visibility curves are represented as a function of the projected baseline $B^{1}$, at a specific wavelength (a $V(B)$ curve). Such a curve represents a number of visibility measurements at different projected baselines, which usually requires the use of multiple telescope pairs and/or moving telescopes.

A new possibility in the $10 \mu \mathrm{m}$ region is the use of spectral dispersion with large wavelength coverage. When using an instrument that has this capability, one can obtain a whole "visibility curve" in one single measurement. Unlike the common $V(B)$ curve, the $V(\lambda)$ curve obtained this way holds many visibility values at only one baseline. The spatial resolution of the observation $(\approx B / \lambda)$ changes by almost a factor of 2 between 7.5 and $14 \mu \mathrm{m}$. Detailed modeling is required to interpret $V(\lambda)$ curves. Most HAe stars show a prominent emission band between 8 and $12 \mu \mathrm{m}$, due to silicate dust. The shape of this emission band varies strongly, depending on chemical composition, particle size and lattice structure of the silicate grains. When simulating interferometric visibilities using disk models, one finds that the detailed shape of the visibility curve depends on the opacities used, i.e. on the dust properties. These are different from star to star, and vary within a disk as a function of distance to the star (van Boekel et al. 2004a). The visibilities measured in the silicate emission feature are a mixture of disk structure and mineralogy. Therefore, in order to deduce information on the disk structure, it is preferable to use measurements at wavelengths outside the silicate feature, which in practice means between about 12 and $13.5 \mu \mathrm{m}$. At $8 \mu \mathrm{m}$, it is also possible to sample the continuum emission, this is however more difficult since here the atmospheric transmission is rather poor.

\subsection{The DD04 disk model}

The disk models used in this work are described in DD04. These are 2D axisymmetric models in which the gas and dust density and temperature are given as a function of radius $R$ and polar coordinate $\Theta$. The disk is assumed to be heated only by

\footnotetext{
${ }^{1}$ The spatial frequency of the observation is: $k=B / \lambda$, where $\lambda$ is the observing wavelength. The units of $k$ are cycles/radian if $\lambda$ and $B$ have the same units.
}

irradiation by the central star. A 2D continuum radiative transfer code is used to compute the entire temperature structure of the disk. The vertical density structure, for a given radial surface density distribution $\Sigma(R)$, is computed by demanding vertical hydrostatic equilibrium. In this way the disk has a selfconsistent temperature and density structure, from which images and SEDs can be computed using a ray-tracer. For this work we use the following stellar parameters: $M_{*}=2.5 M_{\odot}$, $R_{*}=2 R_{\odot}$ and $T_{*}=10000 \mathrm{~K}$, which amounts to a stellar luminosity of $L_{*}=36 L_{\odot}$. All disk models in this work have a disk mass of $0.1 M_{\odot}$, a gas-to-dust ratio of 100 , a surface density distribution of $\Sigma \propto R^{-1.5}$, and an outer radius of $200 \mathrm{AU}$. The DD04 models have an inner disk radius which is calculated self-consistently assuming an optically thick inner rim. The location of the inner rim is set by the dust evaporation temperature, which is about $1500 \mathrm{~K}$ for silicate dust. The CG97 model has an inner radius of $0.21 \mathrm{AU}$, which corresponds to the radius where the black-body temperature is $1500 \mathrm{~K}$. For the dust opacities we use a simple model consisting only of small silicate grains (Laor \& Draine 1993).

If the disk is optically thick enough, the disk has a flaring shape (DD04 and Dullemond 2002). When the optical depth is decreased, a flaring disk can turn into a self-shadowed disk and the SED changes from a group I to a group II shape. The flaring and self shadowed models shown here are the BL1 and the BL4 model from DD04. Both disks have a mass of $0.1 M_{\odot}$. In the BL1 model all the dust mass is in $0.1 \mu \mathrm{m}$ silicate grains. In the BL4 model $99.9 \%$ of the mass has been converted into $2 \mathrm{~mm}$ size grains located in the midplane, while only $0.1 \%$ remains in small $0.1 \mu \mathrm{m}$ grains, thus strongly lowering the opacity of the disk. We stress however, that the flaring vs. selfshadowed behaviour of the disk depends on high vs. lower optical depth, and that dust coagulation is a possible mechanism to achieve lower optical depths.

In the proposed scheme, the outer disk of a self-shadowed source is shielded from direct stellar irradiation by its own inner disk. However, the outer disk receives near-IR radiation emitted by the hot innermost disk regions, and optical/UV radiation which is scattered by the diffuse inner disk atmosphere. Therefore, the temperature and scale height in a self-shadowed disk are still significantly larger than zero. Note that in a flaring disk, there is also a region just outward of the inner rim that is shielded from direct stellar radiation. Contrary to a selfshadowed disk, a flaring disk emerges from the shadow cast by the puffed up inner rim, at distances of a few AU from the star.

\section{Results}

The geometry of the disk models investigated in this work is schematically represented in Fig. 1. The outer disk geometries in the CG97 and DD04 flaring models are very similar, however the inner disk region is different. The DD04 flaring model has a puffed up inner rim, and an intermediate shadowed region (shaded light). The DD04 self-shadowed model has an inner disk structure that is very similar to the DD04 flaring model. The outer disk in the self-shadowed model never rises above the shadow cast by the puffed up inner rim, but is irradiated by the hot inner disk regions. 

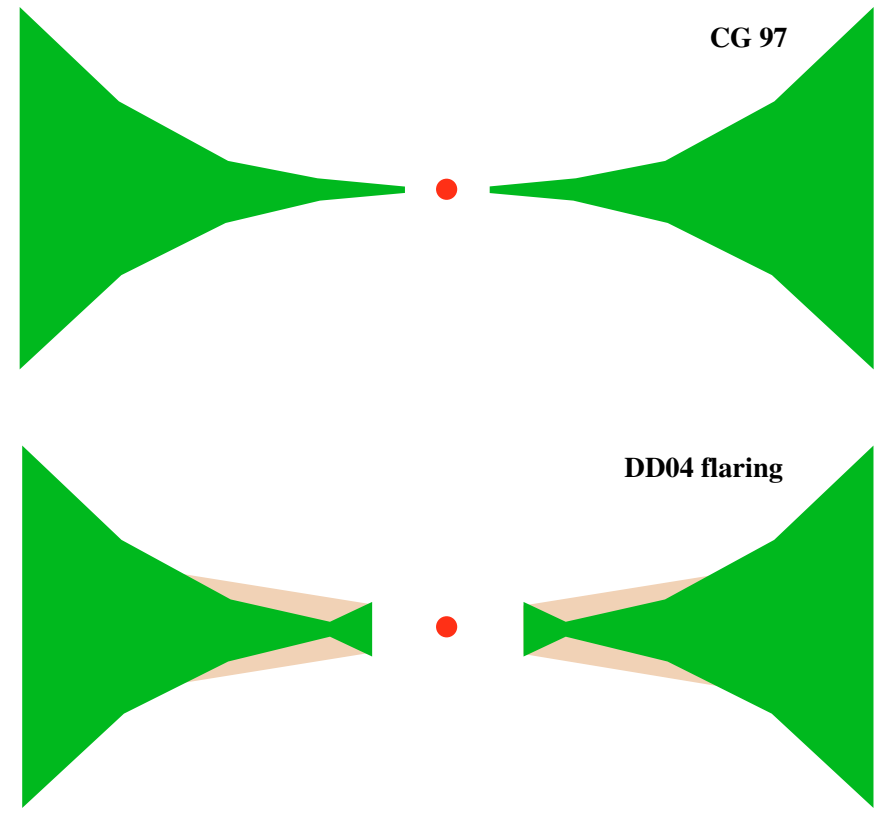

DD04 self-shadowed

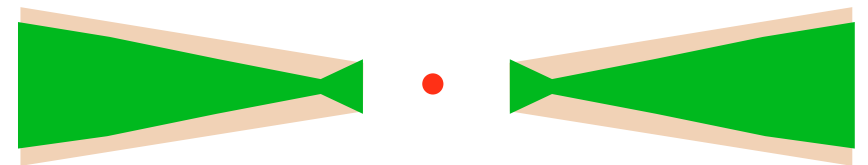

Fig. 1. A schematic representation of the geometries of the disk models studied in this work. The shadow cast by the inner rim is shaded light.

\subsection{Spectral energy distributions}

The emerging spectral energy distributions of the disk models are shown in Fig. 2. The infrared excess emission at far infrared (FIR) wavelengths $(\sim 60-100 \mu \mathrm{m})$ is clearly stronger in the flaring disk models than in the self-shadowed model. The FIR excess is significantly stronger in the DD04 flaring model than in the CG97 (also flaring) model. This can be traced to the simplifications made in the CG97/DDN01 models which do not take properly into account various $3 \mathrm{D}$ radiative transfer effects. In particular, these models do not account for the moderate "boosting" of radiation toward the polar axis to compensate for the occultation in equatorial directions by the disk's own flaring outer regions. In the DD04 models these effects are consistently taken into account by virtue of the full multidimensional radiative transfer treatment used in those models. The near-infrared excess around $2-3 \mu \mathrm{m}$ which is very prominent in the DD04 model SEDs, is much less pronounced in the CG97 model. In the $10 \mu \mathrm{m}$ region, the CG97 and DD04 flaring model SEDs are virtually identical in spectral shape, though the DD04 flaring model has a somewhat higher absolute flux level. The DD04 self-shadowed disk is fainter than the flaring models, and has a bluer continuum slope in the $10 \mu \mathrm{m}$ region.

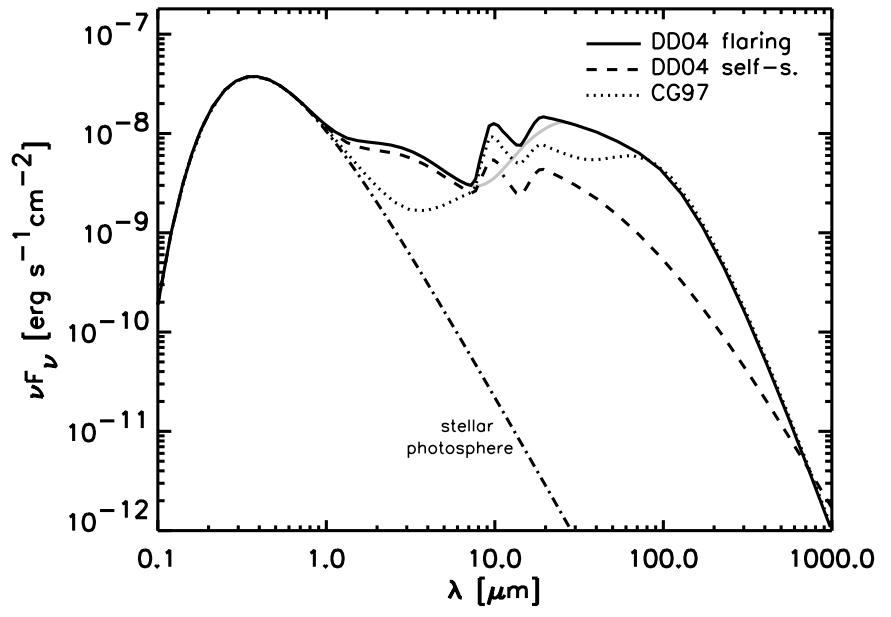

Fig. 2. The spectral energy distributions of the DD04 flaring (full curve), DD04 self-shadowed (dashed curve) and CG97 (dotted curve) models. The full grey curve represents the DD04 flaring model, where the silicate resonances between 8 and $25 \mu \mathrm{m}$ have artificially been removed from the opacity table (see also Sect. 3.3.2 and Fig. 6).

\subsection{Radial intensity profiles}

Figure 3 shows the radial intensity profiles at $12.6 \mu \mathrm{m}$ of a DD04 flaring and self-shadowed disk model, and the CG97 model. Both the DD04 flaring and self-shadowed model essentially exhibit three regimes:

1) the "bright puffed up inner rim", that causes a ringlike emission, contributing mainly between 0.5 and $0.8 \mathrm{AU}$ from the star;

2) a region just behind the inner rim (as seen from the star), where the dust temperatures are much lower than in the inner rim. From this "intermediate shadowed region", relatively little radiation emerges (as can be seen in the cumulative flux distributions of the DD04 models in the lower panel of Fig. 3, which are nearly constant in this region);

3 ) the "outer disk region", whose main flux contribution arises between 3 and $20 \mathrm{AU}$ from the central star. In a flaring disk model, this outer disk region is directly irradiated by the central star.

The "bright puffed up inner rim", "intermediate shadowed region" and "outer disk region" are of course just different parts of the same physical structure, and the distinction made here serves merely to help the reader develop a qualitative understanding of how such geometries translate into interferometric visibilities. In Fig. 3 we have indicated the three regions discussed above. For the DD04 flaring model, the outer radius of the intermediate shadowed region can be well defined to be between 2 and $3 \mathrm{AU}$, where the slope of the cumulative flux distribution clearly increases. In the DD04 self-shadowed model, this radius is less clearly defined but evidently somewhat larger than in the DD04 flaring model. The contribution of the bright inner rim emission to the total system flux depends strongly on wavelength (for the self-shadowed model, the inner rim contributes more than $90 \%$ to the total flux at $6 \mu \mathrm{m}$, about $60 \%$ at $8 \mu \mathrm{m}$, about $35 \%$ at $13 \mu \mathrm{m}$ and less than $5 \%$ at $30 \mu \mathrm{m}$ ), and is 


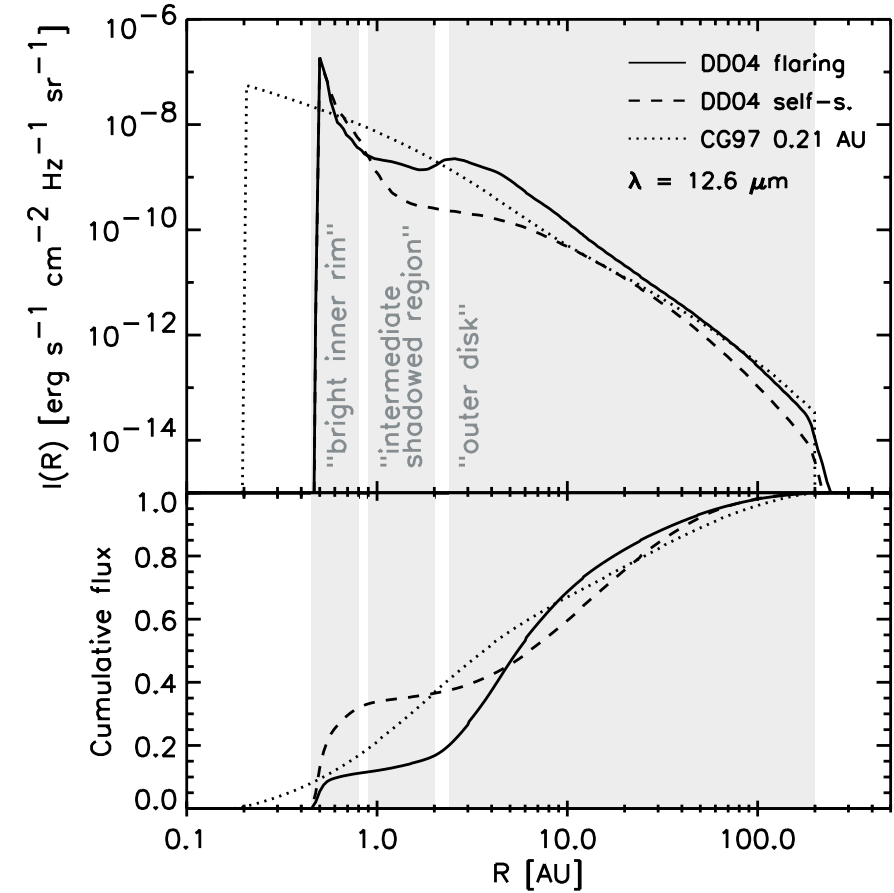

Fig. 3. Radial intensity profiles of the DD04 flaring, DD04 selfshadowed and CG97 models (upper panel). The lower panel shows the normalized cumulative flux distribution of the models. The three regions of the disk that we distinguish ("bright inner rim", "intermediate shadowed region", and "outer disk") are indicated for the DD04 flaring model (in the DD04 self-shadowed model, the intermediate shadowed region extends somewhat further outward).

always higher in a self-shadowed model than in a flaring model harboring the same central star.

The CG97 model has, per definition, a flaring disk structure. Contrary to the DD04 flaring model however, it does not have a bright puffed up inner rim, and consequently it lacks an intermediate shadowed region.

\subsection{Visibility curves}

\subsubsection{Visibility as a function of baseline}

Figure 4 shows the predicted "classical" visibility curves $V(B)$ of the considered disk models, at several wavelengths. From top to bottom we show the predictions for the CG97 model, the DD04 flaring model and the DD04 self-shadowed model. To develop an intuitive understanding of how the characteristics of the emerging intensity distributions of the various models are reflected in their visibility curves, we will discuss the curves in Fig. 4. In this example, the star is put at a distance of $150 \mathrm{pc}$, typical for nearby Herbig stars.

At a baseline of $0 \mathrm{~m}$ all sources are of course unresolved and have a visibility of 1 . The CG97 model shows a steady drop in visibility as the baseline is increased. The slope of the visibility curve changes gradually, reflecting that the radial intensity profile shown in Fig. 3 has no strong substructure. In this sense, the CG97 model is "scaleless".

For the DD04 models, this is different. These models have essentially two scales: the bright inner rim, which emits between 0.5 and $0.8 \mathrm{AU}$, and the outer disk, which emits most of its flux between about 3 and $20 \mathrm{AU}$. In between lies the intermediate shadowed region, whence little flux emerges. This general picture is reflected in the visibility curves. Starting at $0 \mathrm{~m}$, and increasing the baseline, we observe a steady drop in visibility as the outer disk gets more and more resolved. Note that at a baseline of $10 \mathrm{~m}$ the visibility is already significantly lower than 1 , predicting that the largest modern day telescopes might marginally resolve the outer disks in such objects at $10 \mu \mathrm{m}$. For the HAe star HD 100546 this has indeed been observed (Liu et al. 2003; van Boekel et al. 2004b). At a baseline of about $30 \mathrm{~m}$, the outer disk is mostly resolved while the inner rim is still essentially unresolved. Therefore, the visibility curves flatten at this point. The visibility level at this baseline (about $15 \%$ for the flaring model and $40 \%$ for the selfshadowed disk, at $9.8 \mu \mathrm{m}$ ) indicates the fraction of the total system flux that is emitted by the bright inner rim. At longer baselines, the bright inner rim itself becomes resolved by the interferometer, and the visibility gradually goes to its first null. Since the spatial resolution of the interferometer scales inversely with wavelength, whereas the apparent diameter of the inner rim hardly depends on the wavelength, zero visibility is reached first at the shortest wavelengths, and at longer baselines for the longer wavelengths.

\subsubsection{Visibility as a function of wavelength}

Figure 5 shows $V(\lambda)$ curves for the CG97, DD04 flaring and DD04 self-shadowed model, at a number of different baselines. Each of these curves represents a single, dispersed, visibility measurement (the spectral region inaccessible from the ground is shaded grey). Each curve in Fig. 5 can be regarded as a cut through Fig. 4 at a specific baseline, with a much denser wavelength sampling.

The overall trend for all curves is to show the highest visibilities at $8 \mu \mathrm{m}$, and lower visibilities at $13 \mu \mathrm{m}$. This is because the apparent size of the disks increases with wavelength more rapidly than the interferometric resolution decreases. There is generally a sharp decrease in visibility between 8 and $10 \mu \mathrm{m}$. There are two reasons for this. For the models with an inner rim one reason is that the emission from this rim dominates the spectrum below about $8 \mu \mathrm{m}$. The emission at $10 \mu \mathrm{m}$ originates from more extended regions of the disk, resulting naturally in a lower visibility than the $8 \mu \mathrm{m}$ emission. A second reason for the decline of the visibility between 8 and $10 \mu \mathrm{m}-$ and for the gentle rise in visibility toward $13 \mu \mathrm{m}$ in some models - is that the flux in the $10 \mu \mathrm{m}$ silicate feature originates predominantly from the warm surface layers of the disk, while the flux outside the feature comes from the cooler regions below. The warm dust in the surface layer can radiate in the mid-infrared out to larger radii than the cooler dust in the disk interior. In other words: in the warm surface layers the Wien exponential cut-off in the mid-infrared takes effect at larger radii than in the disk interior. This explanation also holds for the CG97 models, which do not have an inner rim.

In Fig. 6 we demonstrate the importance of the silicate resonance for the simulated visibility curves. We show the 


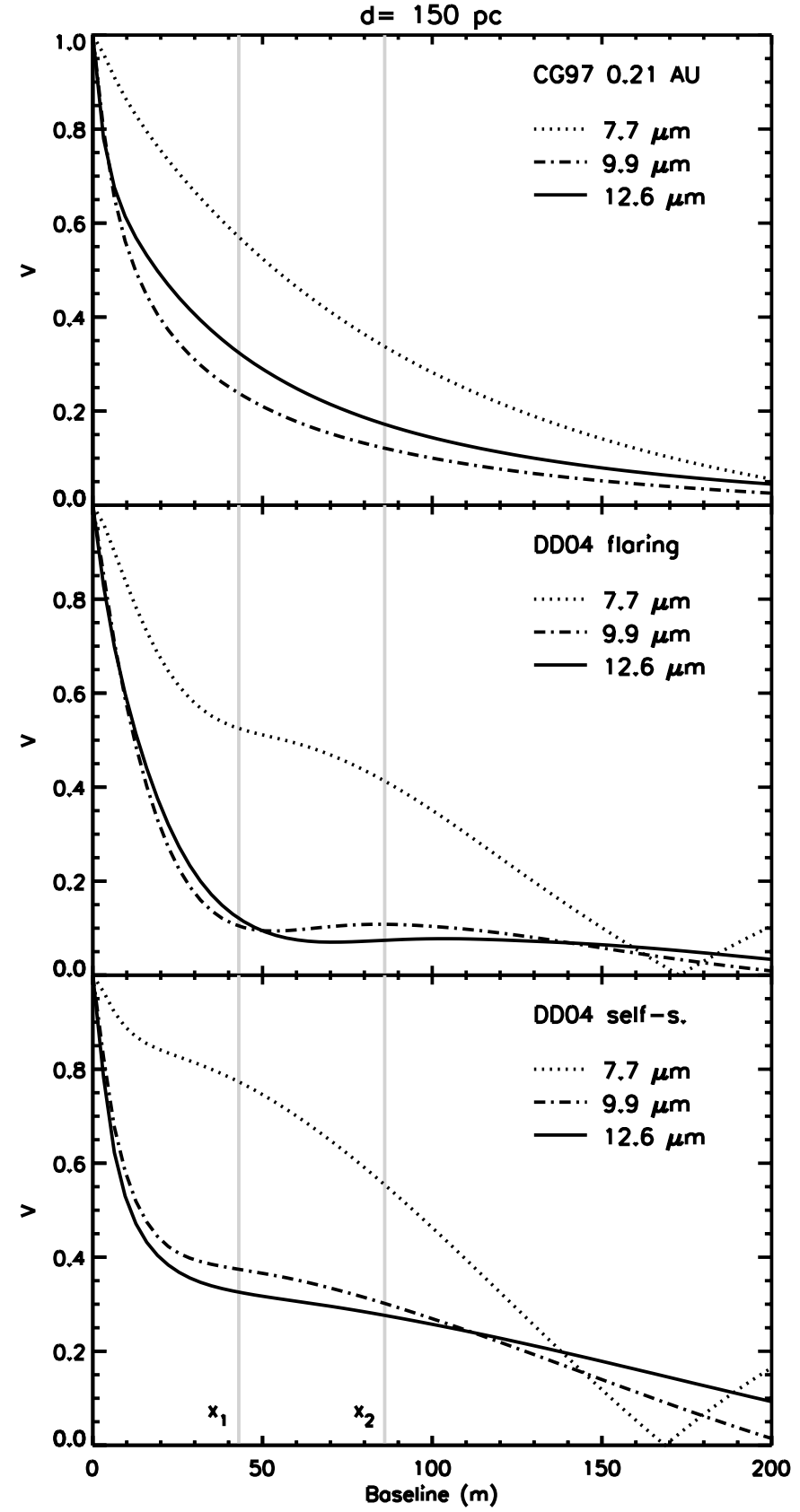

Fig. 4. Simulated visibility curves $V(B)$ of a CG97, a DD04 flaring, and a DD04 self-shadowed disk model (from top to bottom, for $V(\lambda)$ curves of these models see Fig. 5). We show model visibilities at three different wavelengths. $x_{1}$ and $x_{2}$ denote optimum baselines to distinguish between the various models (see Sect. 3.4).

visibilities of the DD04 flaring model (full curves, see also the middle panel of Fig. 5). To calculate visibility curves of a model without silicate resonances, we removed the 10 and 20 micron silicate features from the opacity table prior to the ray tracing (dotted curves). The large influence of the opacity of the material on the resulting visibilities is evident. The SED of the model without silicate resonances is indicated by the full grey curve in Fig. 2.

The interpretation of the curves in Fig. 5 in terms of disk geometry is not straightforward, for several reasons. First, the

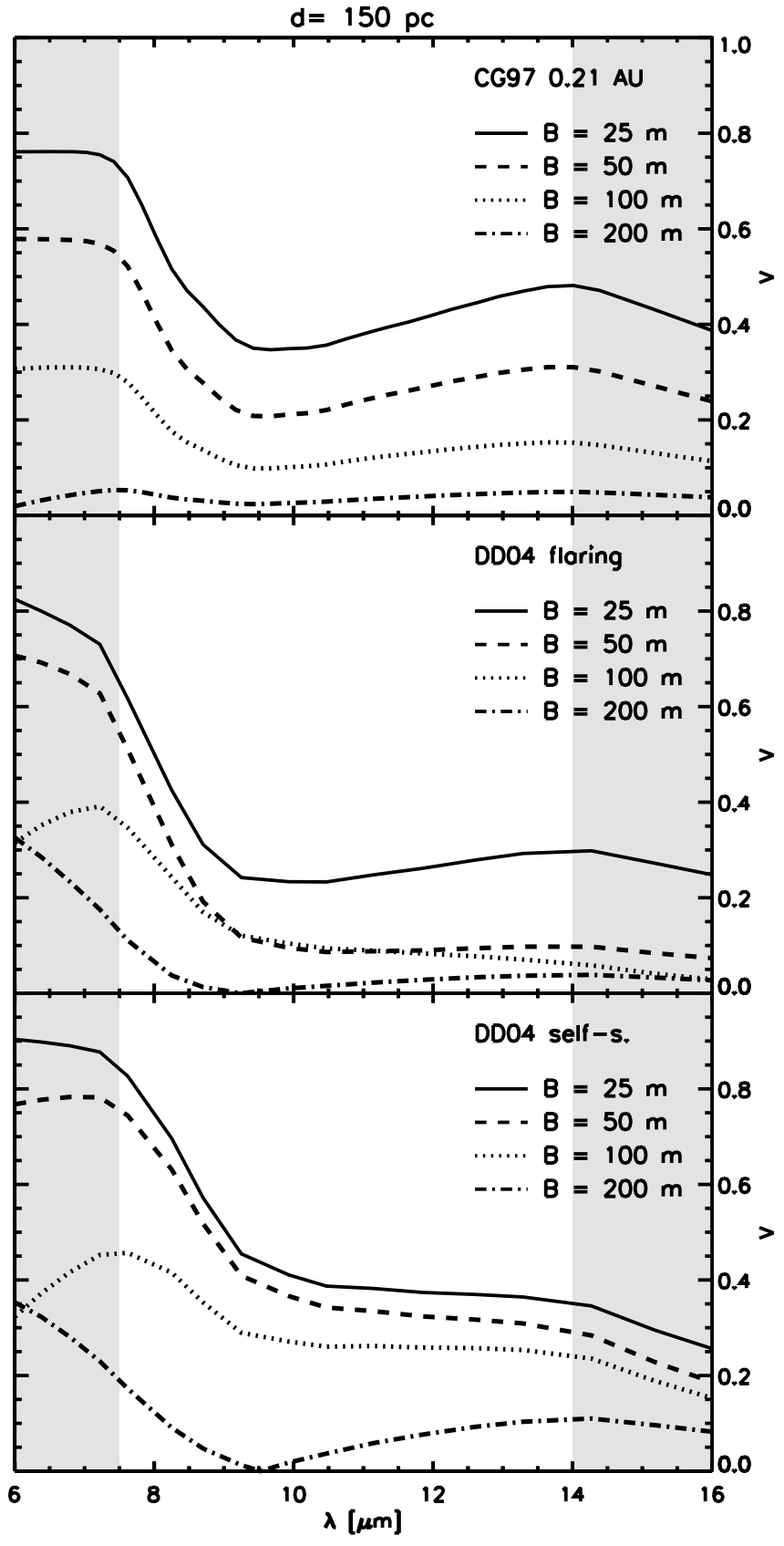

Fig. 5. Visibility curves $V(\lambda)$ of a CG97, a DD04 flaring, and a DD04 self-shadowed disk model (for $V(B)$ curves of these models see Fig. 4). We show the predicted behaviour of the visibility as a function of wavelength $V(\lambda)$, for several different baselines. The wavelength regions that are inaccessible from the ground are shaded grey.

emerging intensity distribution of the disk changes with wavelength. Second, the spatial resolution of the interferometer decreases by almost a factor of two between the short and long wavelength edges of the $10 \mu \mathrm{m}$ atmospheric window ( $N$-band). Third, how the disk intensity distribution (and thus the visibilities) changes with wavelength depends on the opacity of the dust, i.e. on mineralogy (Fig. 6). When we measure a single $V(\lambda)$ curve, what we see therefore is a mixture of disk geometry changing with wavelength, instrumental resolution changing with wavelength, and the mineralogy of the source. 


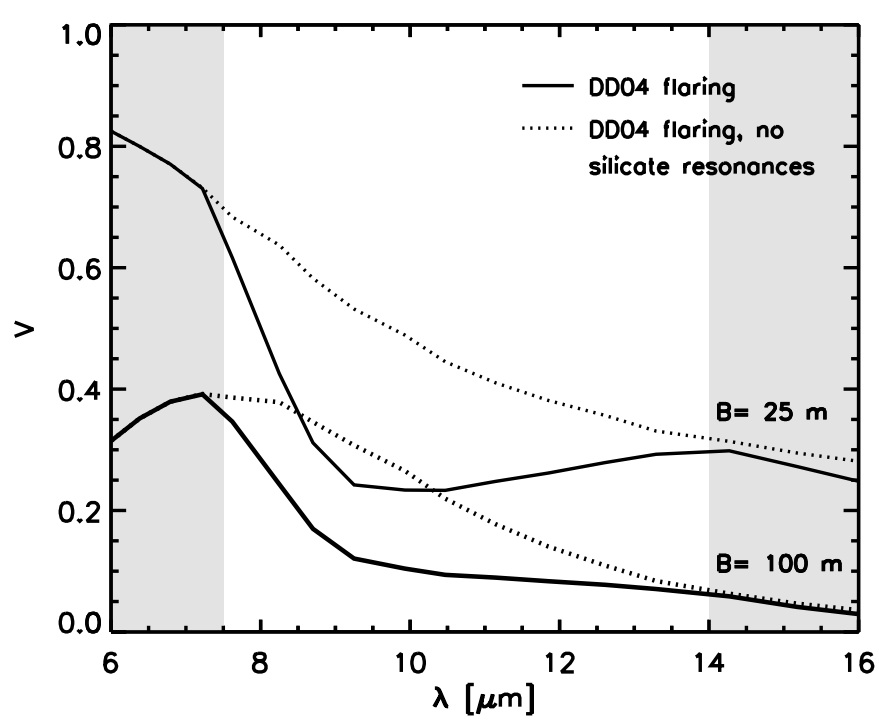

Fig. 6. The effect of the increased dust opacity in the silicate feature on the visibilities. The full lines show the visibility curves of the DD04 flaring model, the dashed lines show the visibility curves of the same model, where the silicate resonances have been removed in the opacities prior to the ray tracing.

As the mineralogy changes from star to star, it is difficult to obtain general diagnostics for disk geometry from a $V(\lambda)$ curve. The detailed interpretation of such measurements requires a model of each individual star, where both the spectrum (mineralogy) and the disk structure are fitted simultaneously. As a general diagnostic for disk structure it is thus preferable to measure visibilities in the continuum, where the visibilities do not depend strongly on mineralogy. We will come back to this in Sect. 3.4.

However, the spectral capabilities of the interferometer develop their full strength, when one uses observations at several baselines in order to reconstruct an "image" of the disk ${ }^{2}$. With spectrally dispersed visibilities, the spectrum of the disk is then known immediately at all positions in the disk. It is then possible to study the mineralogy, size distribution and chemical composition of dust grains in the disk surface layer as a function of distance to the central star, providing crucial information about dust processing and radial mixing in disks.

If the intensity distribution in the disk is strongly centrally peaked like the models discussed in the present paper, the correlated flux obtained at a single, long baseline ${ }^{3}$ can be directly interpreted as the spectrum of the innermost regions of the disk (in the correlated spectrum obtained with only one measurement, there is still an unknown spatial term mixed in, that typically introduces a slope in the spectrum. This however has little

\footnotetext{
${ }^{2}$ In practice this will be easiest for disks that are seen not too far from pole on, so their image has a high degree of azimuthal symmetry. This limits the number of baselines needed, and requires the measurement of visibility amplitude only, since all phases will be approximately 0 .

3 The visibility is the ratio of the correlated flux and the total flux $\left(F_{\text {cor }} / F_{\text {tot }}\right)$. The correlated flux, or correlated spectrum in the case of a spectrally dispersed measurement, is the quantity an interferometer measures.
}

influence on the derived mineralogy). The outer disk spectrum can then be obtained as a difference between the integrated disk spectrum and the inner disk spectrum. Applying this method to the first spectrally resolved full $N$-band visibility measurements of HAe stars, it was demonstrated by van Boekel et al. (2004a) that the mineralogy in the disk can vary strongly with distance to the star.

\subsection{Distinguishing between the various models}

The goal of this study is to show how interferometric measurements can be used to distinguish between the various disk models. Clearly, the curves in Fig. 4 are different for the different models. However, one will typically not have continuous measured visibility curves at hand, but rather have samples at a few different baselines. Here we show that it is, at least in principle, possible to distinguish both between the CG97 and DD04 models on one hand, and between the DD04 flaring and DD04 self-shadowed on the other, using measurements at only two appropriately chosen baselines.

The distinction between the CG97 and DD04 models is based on the absence of an intermediate shadowed region in the former. At spatial scales corresponding to the intermediate shadowed region in the DD04 models, little flux emerges. Therefore, the visibility curves are relatively flat at the baselines corresponding to these spatial scales, they show a "plateau" (very prominent in the DD04 flaring model visibility at $12.6 \mu \mathrm{m}$ in Fig. 4). The CG97 model does not have such specific spatial scales with much reduced emergent intensity, and therefore lacks the plateau in the visibility curve. The visibility curves of CG97 and DD04 models thus have a different slope at baselines corresponding to the scale of the intermediate shadowed region. Once this slope difference has been detected, DD04 flaring and DD04 self-shadowded disks can be distinguished by the relative contribution of the bright inner rim to the total system flux, which is much higher for a self-shadowed model. Note that the bright inner rim itself is virtually identical in both models, but the outer disk is much brighter in the flaring disk than in the self-shadowed case. Therefore, the predicted visibilities at our selected baselines are much lower for the flaring model. We recall that deducing properties about the disk structure is best done outside the silicate emission feature, which in practice favours the region between 12 and $13 \mu \mathrm{m}$.

A measurement at a specific baseline samples the corresponding angular scale, and the physical scale (in AU) associated with this baseline therefore depends linearly on the distance to the star. It is therefore convenient to introduce the "normalized baseline"

$x \equiv \frac{B}{D}$

where $B$ is the baseline in meter and $D$ is the distance to the star in parsec. Consider the emission of a small part of the disk, arising between $R$ and $R+\mathrm{d} R$ from the central star, i.e. a ring with angular diameter $\theta=2 R / d$ (where $d$ is the distance to the star). The visibility curve of such an annulus of emission is the zeroth order Bessel function:

$V_{\mathrm{an}}(B)=J_{0}\left(\frac{\theta B}{\lambda}\right)$ 


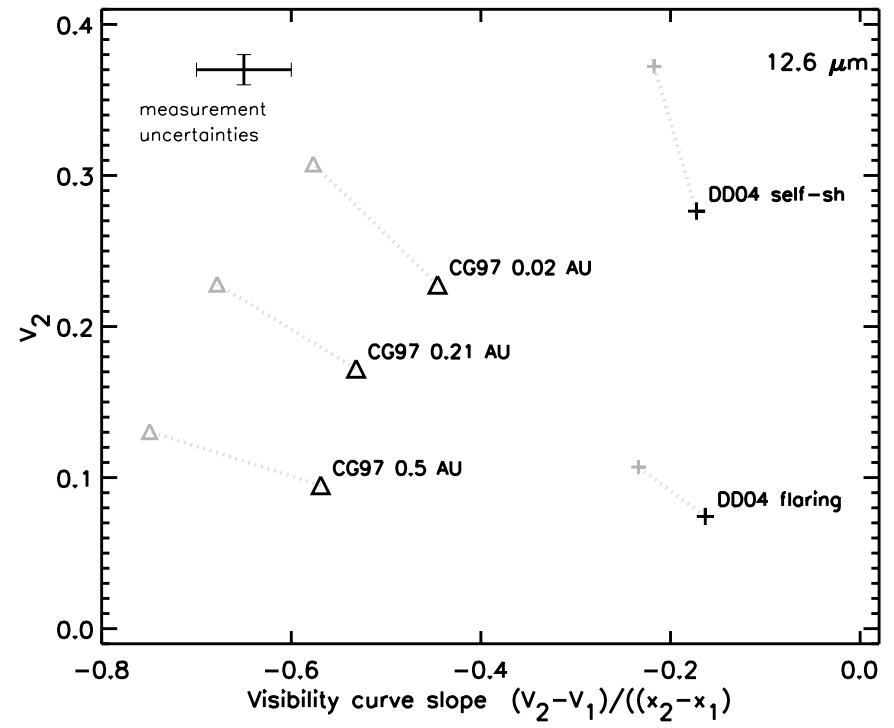

Fig. 7. A diagnostic diagram used to distinguish between models with (DD04) and without (CG97) a bright inner rim, and between models with a flaring (DD04 flaring) and self-shadowed (DD04 self-sh) outer disk geometry. On the horizontal axis we plot the slope of the visibility curves between two appropriately chosen "normalized" baseline lengths $x_{1}$ and $x_{2}\left(x_{i}=B_{i} / d\right.$, where $B_{i}$ is the baseline in $\mathrm{m}$ and $d$ is the distance to the star in pc, see Sect. 3.4 for how $x_{1}$ and $x_{2}$ are best chosen). On the vertical axis we plot the predicted visibility at the longest baseline. The CG97 models have a much steeper slope than the DD04 models. The DD04 self-shadowed model has a much higher visibility than the DD04 flaring model. In the upper left corner we have indicated the uncertainties due to the limited precision of the visibility measurements, where we have assumed a $1 \%$ accuracy in visibility. In grey symbols we have plotted where in the diagram the various models end up if we artificially remove the silicate feature (as we did in Fig. 6). This can be regarded as an upper limit for the uncertainty due to mineralogy.

where $B$ is the interferometric baseline, and $\lambda$ is the wavelength of observation. The visibility reaches the first null at a baseline of about

$B_{0} \approx 158 \frac{\lambda_{\mu \mathrm{m}}}{\theta_{\mathrm{mas}}}[\mathrm{m}]$

where for convenience the wavelength and annulus angular diameter have been expressed in $\mu \mathrm{m}$ and milli-arcseconds, respectively. Our goal is to detect the effect of the intermediate shadowed region, i.e. the very low flux contribution from annuli between about 0.8 and $3 \mathrm{AU}$. To estimate which baseline is most sensitive to emission from an annulus with diameter $\theta$, let us take the baseline where the visibility has half its maximum value, $V_{\text {an }}=0.5$ :

$B_{0.5} \approx 100 \frac{\lambda_{\mu \mathrm{m}}}{\theta_{\mathrm{mas}}}[\mathrm{m}]$.

For an annulus of $2 \mathrm{AU}$ radius, this corresponds to a normalized baseline of $x=\lambda_{\mu \mathrm{m}} / 40$. Let us take the region between 1 and $2 \mathrm{AU}$ from the star as characteristic for the intermediate shadowed region.

We find that in order to most clearly separate the specific DD04 models (with an intermediate shadowed region) used in this work from the CG97 models (without an intermediate shadowed region), the best choice for $x$ is:

$x_{1}=0.038\left(\frac{\lambda}{10 \mu \mathrm{m}}\right)\left(\frac{L_{*}}{L_{\odot}}\right)^{\frac{1}{2}}\left[\frac{\mathrm{m}}{\mathrm{pc}}\right]$

$x_{2}=2 x_{1}$.

To allow the above formula to be applied to stars of different luminosity we have applied a simple scaling of $x$ with the square root of the luminosity. The visibility is now sampled at the baselines corresponding to $x_{1}$ and $x_{2}$ (for our $36 L_{\odot}$ star at $150 \mathrm{pc}$ and at a wavelength of $12.6 \mu \mathrm{m}$ these are 43 and $86 \mathrm{~m}$, respectively, see also Fig. 4), yielding visibilities $V_{1}$ and $V_{2}$. In Fig. 7 we plot the on the vertical axis the visibility measured on the longest of the two baselines $\left(V_{2}\right)$. On the horizontal axis we plot the deduced slope of the visibility curve between $x_{1}$ and $x_{2}$. In addition to the CG97 model with an inner radius of $0.21 \mathrm{AU}$ we plot CG97 models with inner radii of 0.02 and $0.5 \mathrm{AU}$, to illustrate the behaviour with varying inner radius. From this figure we see that:

1) the CG97 models have a much steeper slope than the DD04 models in this baseline regime;

2) the DD04 flaring model has a much lower visibility at the long baseline than the DD04 self-shadowed model (and on the short baseline as well).

We can conclude therefore, that it is possible to discriminate between the various models, with a very limited number of measurements. In the upper left corner of Fig. 7 we indicate the uncertainty in the measured location of a source in this diagram due to limited precision of the visibility measurments. We have here assumed a visibility accuracy of $1 \%$, which is what MIDI aims to achieve. The difference in the position of the models with and without a silicate feature (plotted with black and grey symbols, respectively) can be regarded as the extreme case for the uncertainty arising from mineralogy, since for each individual source, we know what the silicate feature looks like.

This analysis has been done in an idealized world where we have both assumed that the disks are pole-on, and that our models are a good representation of the true disk geometry. The optimum choice for $x_{1}$ and $x_{2}$ depends on the geometry of the disk and may therefore in reality be somewhat different than the values given in our recipe (Eq. (5)). If the outer disk is smaller than we predict, the value of $x_{1}$ should be increased. If the bright inner rim is located at radii somewhat larger than predicted, the value of $x_{2}$ should be decreased. There is however evidence that the bright inner rim is located at radii somewhat smaller than predicted in our models (Eisner et al. 2003), and therefore this is not likely a reason for concern. In practice, measurements at more than two baselines $(\approx 5)$ are probably needed to unambiguously establish the nature of the sources.

\section{Conclusions}

We have presented model calculations of interferometric visibilities of circumstellar disks around Herbig Ae stars. We compare predictions for disks with (DD04) and without a bright inner rim (CG97). We show that it is possible to distinguish 
between both model possibilities using a small number of interferometric measurements in the $10 \mu \mathrm{m}$ atmospheric window. Such measurements also allow to distinguish between flaring and self-shadowed disk models. This allows testing of the hypothesis that group I and group II sources correspond to flaring and self-shadowed disks, as is suggested by their spectral energy distributions.

Acknowledgements. R. Lachaume and L. B. F. M. Waters are gratefully acknowleged for constructive comments on the manuscript.

\section{References}

Akeson, R. L., Walker, C. H., Wood, K., et al. 2005, ApJ, 622, 440 Augereau, J. C., Lagrange, A. M., Mouillet, D., \& Ménard, F. 2001, A\&A, 365, 78

Chiang, E. I., \& Goldreich, P. 1997, ApJ, 490, 368

Dominik, C., Dullemond, C. P., Waters, L. B. F. M., \& Walch, S. 2003, A\&A, 398, 607

Dullemond, C. P. 2002, A\&A, 395, 853

Dullemond, C. P., \& Dominik, C. 2004a, A\&A, 417, 159

Dullemond, C. P., \& Dominik, C. 2004b, A\&A, 421, 1075

Dullemond, C. P., Dominik, C., \& Natta, A. 2001, ApJ, 560, 957

Eisner, J. A., Lane, B. F., Akeson, R. L., Hillenbrand, L. A., \& Sargent, A. I. 2003, ApJ, 588, 360

Eisner, J. A., Lane, B. F., Hillenbrand, L. A., Akeson, R. L., \& Sargent, A. I. 2004, ApJ, 613, 1049

Eisner, J. A., Hillenbrand, L. A., White, R. J., Akeson, R. L., \& Sargent, A. I. 2005, ApJ, 623, 952
Grady, C. A., Polomski, E. F., Henning, T., et al. 2001, AJ, 122, 3396 Grady, C. A., Woodgate, B., Torres, C. A. O., et al. 2004, ApJ, 608, 809

Herbig, G. H. 1960, ApJS, 4, 337

Isella, A., \& Natta, A. 2005, in press

Kenyon, S. J., \& Hartmann, L. 1987, ApJ, 323, 714

Lachaume, R., Malbet, F., \& Monin, J.-L. 2003, A\&A, 400, 185

Laor, A., \& Draine, B. T. 1993, ApJ, 402, 441

Lawson, P. R. 2000, Principles of Long Baseline Stellar Interferometry Leinert, C., van Boekel, R., Waters, L. B. F. M., et al. 2004, A\&A, 423, 537

Liu, W. M., Hinz, P. M., Meyer, M. R., et al. 2003, ApJ, 598, L111

Mannings, V., \& Sargent, A. I. 1997, ApJ, 490, 792

Meeus, G., Waters, L. B. F. M., Bouwman, J., et al. 2001, A\&A, 365, 476

Millan-Gabet, R., Schloerb, F. P., \& Traub, W. A. 2001, ApJ, 546, 358

Monnier, J. D., Millan-Gabet, R., Billmeier, R., et al. 2005, ApJ, 624, 832

Natta, A., Grinin, V., \& Mannings, V. 2000, Protostars and Planets IV, 559

Natta, A., Prusti, T., Neri, R., et al. 2001, A\&A, 371, 186

Skinner, S. L., Brown, A., \& Stewart, R. T. 1993, ApJS, 87, 217

Tuthill, P. G., Monnier, J. D., \& Danchi, W. C. 2001, Nature, 409, 1012

van Boekel, R., Min, M., Leinert, C., et al. 2004a, Nature, 432, 479

van Boekel, R., Waters, L. B. F. M., Dominik, C., et al. 2004b, A\&A, 418, 177

Vink, J. S., Drew, J. E., Harries, T. J., \& Oudmaijer, R. D. 2002, MNRAS, 337, 356 